\title{
Roles of N6-Methyladenosine Demethylase FTO in Malignant Tumors Progression
}

\author{
Qing-Kang Zheng' \\ Chao $\mathrm{Ma}^{\prime}$ \\ Irfan ullah ${ }^{2}$ \\ Kang $\mathrm{Hu}^{\prime}$ \\ Rui-Jie $\mathrm{Ma}^{3}$ \\ Nan Zhang ${ }^{4}$ \\ Zhi-Gang Sun (iD) ${ }^{5}$
}

'School of Clinical Medicine, Weifang Medical University, Weifang, People's Republic of China; ${ }^{2}$ Department of Surgery, Khyber Medical University Peshawar, Peshawar, Pakistan; ${ }^{3}$ Cheeloo College of Medicine, Shandong University, Jinan, People's Republic of China; ${ }^{4}$ Department of Oncology, Central Hospital Affiliated to Shandong First Medical University, Jinan, People's Republic of China; ${ }^{5}$ Department of Thoracic Surgery, Central Hospital Affiliated to Shandong First Medical University, Jinan, People's Republic of China
Correspondence: Zhi-Gang Sun

Department of Thoracic Surgery, Central Hospital Affiliated to Shandong First

Medical University, Jinan, 2500 I3, People's

Republic of China

Tel +86I3370582825

Email sunszg@।26.com

Nan Zhang

Department of Oncology, Central

Hospital Affiliated to Shandong First

Medical University, Jinan, 2500 I3, People's

Republic of China

Tel +86I3370582850

Email zlkzn20I6@I26.com

\begin{abstract}
In 2007, the fat mass and obesity-associated (FTO) gene was discovered initially to regulate body mass index and obesity and was subsequently found to be the first mRNA N6methyladenosine (m6A) demethylation enzyme, which can demethylate m6A. A growing body of evidence shows that $\mathrm{m} 6 \mathrm{~A}$ modification is involved in a variety of cell biological processes, including cell proliferation, apoptosis, and self-renewal through different regulatory mechanisms. In recent years, a large number of studies have found that m6A modification play key role in the occurrence and development of tumors, such as acute myeloid leukemia, breast cancer, lung cancer, etc. As a function of m6A demethylase, FTO has attracted more and more attention in cancer. There is evidence that specific FTO single nucleotide polymorphisms (SNPs) may be significantly associated with overweight and cancer susceptibility by regulating the expression of related genes. Besides, when the expression level of FTO is altered or dysfunctional, it may be involved in the occurrence and progression of a variety of tumors as a tumor suppressor gene or oncogene, usually in an m6A-dependent manner. Further research found that FTO is involved in the development of different kinds of malignant tumors, but the mechanism is unknown. According to this review, The FTO gene's research progress in tumors is reviewed, aiming to find new targets for molecular pathological diagnosis and molecular targeted therapy of tumors. Keywords: FTO, cancers, N6-methyladenosine, SNPs, FTO inhibitors
\end{abstract}

\section{Introduction}

In 1999, the fat mass and obesity-associated gene were originally cloned in fusion toe mutant mice by exon tapping analysis and was named "Fatso", whose function was unknown. ${ }^{1}$ Then, subsequent evidence has shown that FTO had the effects of promoting fat formation and obesity. ${ }^{2,3}$ In recent years, the prevalence of obesity and cancer has been on the rise, and the research on the association between the two has raised great interest among scientists. Epidemiological studies have also shown that FTO SNPs and obesity play a significant role in tumor progression. Recent studies have shown that m6A modifications are widely and complex in eukaryotes and are involved in cancer progression under multiple regulatory mechanisms. FTO acts as a messenger RNA N6methyladenosine (m6A) demethylase, which has been shown to play an essential role in tumorigenesis in an m6A-dependent manner. Therefore, to find new targets for tumor therapy, studies on the role of FTO as m6A demethylation enzyme of mRNA in related tumor pathways are increasing, and some achievements have been made.

\section{The FTO Gene}

In 2007, researchers identified a $1.6-\mathrm{Mb}$ deletion on chromosome 8 in a mouse mutant fusion toe, including three genes with unrevealed function (FTS, FTM, and 
FTO). ${ }^{4}$ Through a genome-wide association study, researchers identified a group of single nucleotide polymorphisms (SNPs) closely associated with human obesity in the intron region of the FTO gene, which has since been formally named fat mass and obesity-associated protein (FTO). The total length of the FTO gene is $410.50 \mathrm{~KB}$, located on chromosome 16q12.2, and contains 8 introns and 9 exons. FTO is widely expressed in adipose tissue and skeletal muscle of human tissues, and its high expression in the hypothalamus indicates that it may play a pivotal role in regulating appetite and energy metabolism. ${ }^{5}$ FTO gene is only found in vertebrates and algae but not expressed in plants, fungi, or invertebrates. ${ }^{6}$ More importantly, FTO is mainly located in the nucleus and can partially shuttle between the nucleus and cytoplasm through mechanisms mediated by the Exportin 2 (XPO2) family, thus playing an essential role in the regulation of a variety of cellular biological processes. ${ }^{7}$

\section{The Structure and Substrate of FTO}

The FTO gene belongs to the ALKB family of 2-oxoglutarate and $\mathrm{Fe}$ (II)-dependent dioxygenase proteins and has been identified as a DNA/RNA demethylase that demethylates 3-methyluracil (3-Meu) in single-stranded RNA and 3-methylthymine (3-Met) in single-stranded DNA. ${ }^{8,9}$ By studying the crystal structure of FTO, Han et $\mathrm{al}^{10}$ found that there is an extra loop on one side of the conserved jelly roll motif in the FTO structure, which is conducive to competitive binding of FTO with unmethylated doublestranded DNA.

Chemical modification greatly enriched the function and genetic diversity of DNA by removing or introducing various groups. For instance, N6-methyladenosine (m6A) is the most plentiful mRNA modification in eukaryotes, which plays a critical role in mRNA stability, translation, and selective splicing. ${ }^{11-13}$ As the first discovered mRNA m6A demethylase, FTO can effectively demethylate the target gene m6A in vivo and in vitro, thus affecting the function of the target protein. ${ }^{14}$ Mechanically, m6A methylation was found to be a dynamic reversible process consisting of methyltransferases complex(writers), demethylases (erasers), and RNA-binding proteins (readers). ${ }^{15-17}$ Writers are processes that mediate the methylation of RNA m6A, including METTL3, METTL14, and WTAP. Readers are responsible for recognizing the methylation information of RNA m6A and regulating gene expression, including YTH domain proteins and IGF2BPs, by improving the efficiency of mRNA translation. ${ }^{18}$ Erasers mediate the demethylation of RNA, such as FTO and ALKBH5. The m6A modification plays a vital role in post-transcriptional gene expression by adding methyl groups to RNA by the Writers and recognized by various readers. Besides, the m6A modification process became dynamic and reversible by erasers and thus played a role in regulating multiple genes' expression. ${ }^{12,13,19}$ (Figure 1).

However, Mauer et al found that FTO preferentially demethylates N6,2'-O-dimethyladenosine (m6Am) rather than $\mathrm{m} 6 \mathrm{~A}$, and that m6Am was more likely to stabilize mRNA. ${ }^{20}$ These results indicated that the demethylation activity of FTO to m6Am was significantly higher than that of $\mathrm{m} 6 \mathrm{~A}$ and that $\mathrm{m} 6 \mathrm{Am}$ was more sensitive to changes in the depletion of FTO. However, another study found that FTO binds to various RNAs, including mRNA, tRNA, and snRNA. They found that the total amount of m6A in different cell lines was about ten times higher than that of m6Am so that m6A demethylation may be dominant. ${ }^{21}$

Zhang et al also further confirmed that FTO was indeed the demethylase of m6Am, but the overall content of m6Am was much lower than that of m6A. ${ }^{22}$ Although the distribution of FTO in the nucleus and cytoplasm is different, $\mathrm{m} 6 \mathrm{~A}$ is its dominant substrate.

\section{Single Nucleotide Polymorphism (SNPs) in FTO and Cancer Susceptibility}

Single nucleotide polymorphism (SNP), refers to the DNA sequence polymorphism caused by the variation of a single nucleotide at the genome level, is the most common form of heritable variation in humans. For instance, due to genome-wide association studies (GWAS), multiple single nucleotide polymorphisms (SNPs) on intron 1 of FTO were found to be significantly associated with the risk of obesity and the occurrence of certain tumors. ${ }^{2,23}$ Also, the relationship between increased FTO expression and SNP risk genotypes has been demonstrated in human blood cells and fibroblasts. ${ }^{24,25}$ Studies have shown that FTO SNPs are positively correlated with an increased risk of some cancers, indicating that FTO plays a role in cancer pathogenesis. ${ }^{26}$ For example, multiple SNPs in the FTO intron 1 region exerted an important role in breast cancer, such as rs7206790, rs9939609, rs8047395, and rs1477196, but rs1477196 showed the strongest association. ${ }^{27}$ Rs16953002 and rs12596638, located in FTO intron 8, have been shown to play a positive regulatory role in the 


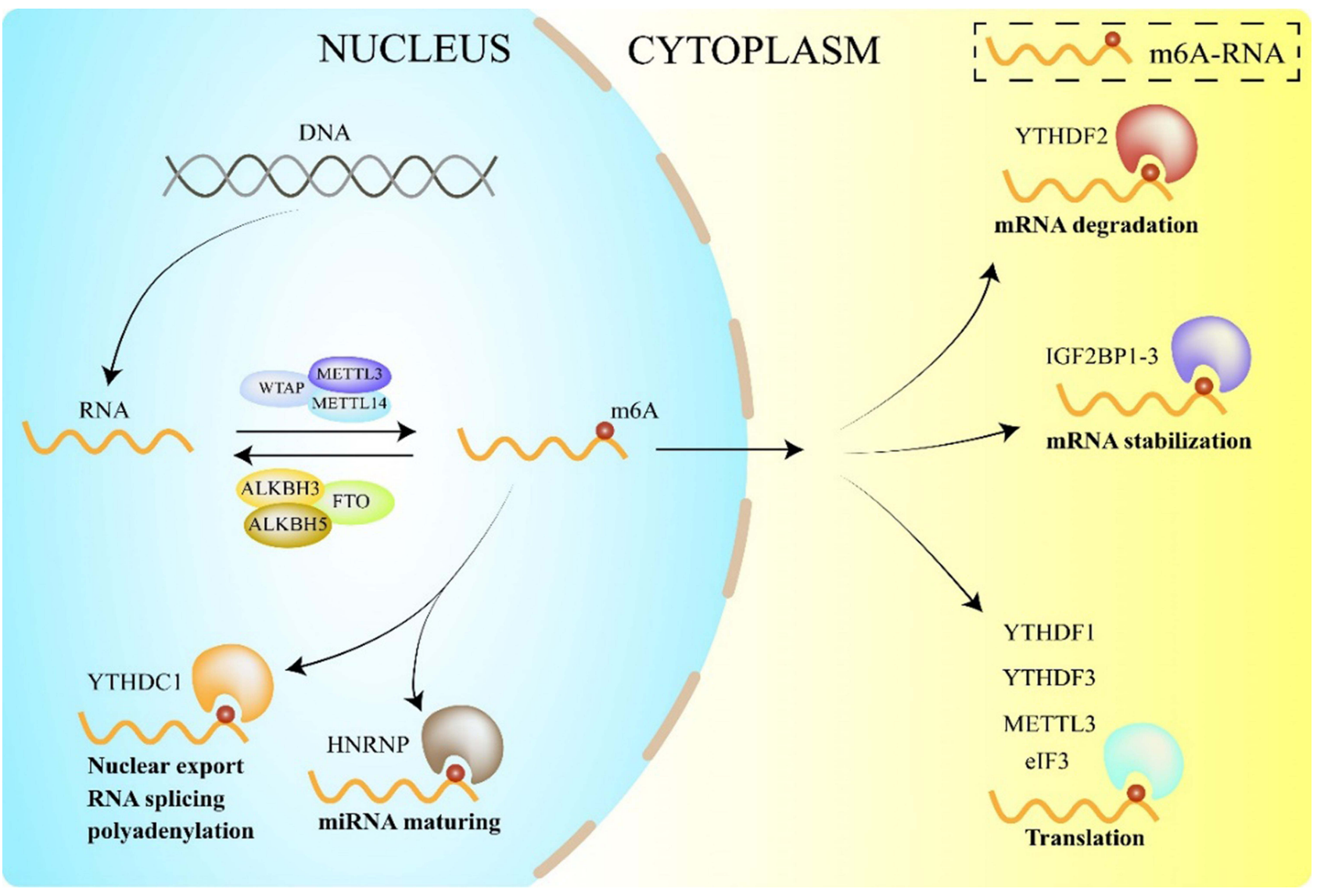

Figure I m6A dynamic regulation and FTO's functions.

occurrence of melanoma. ${ }^{28}$ A retrospective analysis showed that rs9939609 polymorphism was significantly associated with cancer risk in Asian populations and was particularly predictive of early diagnosis of pancreatic and endometrial cancers. ${ }^{29}$ Currently, studies have found that FTO gene polymorphism can not only regulate FTO gene expression and transcription factor binding region but also regulate related adjacent genes (such as IRX3, RPGRIP1L, RBL2, IRX5) to participate in the progress of cancer through various ways. ${ }^{30}$ Given that cancer is always multifactorial, the relationship between FTO SNPs and cancer is complex and variable. Although gene expression regulated by some FTO polymorphisms is associated with cancer susceptibility, further research is needed to explore the specific mechanisms.

\section{Biological Activity of the FTO in Cancers}

Although the research on the relationship between FTO and cancers and the mechanism of action was still in the early stage, more and more shreds of evidence showed that FTO was significantly over-expressed in a variety of tumor tissues and was highly correlated with the prognosis of tumors. With the development of research, the role of m6A modification in a variety of cancers has been increasingly confirmed. As an m6A demethylase, FTO was overexpressed in a variety of cancer tissues and played the role of oncogene or tumor suppressor gene in an m6A-dependent manner, participating in the regulation of tumor progression.

\section{Overexpression of FTO Results in Cellular Proliferation and Apoptosis Inhibition}

Messenger RNA carries the genetic information between DNA and proteins, and its potential mechanisms have been extensively investigated. It has been reported that m6A modification is one of the most classical internal modifications in mRNA, which can reduce mRNA's stability and lead to its degradation. FTO can demethylate m6A and increase the stability of mRNA in related tumor pathways, promoting tumor progression. Li et al ${ }^{31}$ analyzed the whole genome of AML patients and found that FTO was 
highly expressed in specific AML subtypes, such as $\mathrm{t}$ (11q23)/MLL rearrangement, $\mathrm{t}$ (15;17)/PML-RARA, FLT3-ITD, and/or NPM1 mutations. Overexpression of FTO significantly promoted the viability and proliferation of human AML cells, inhibited the differentiation and apoptosis of human AML cells, and promoted the occurrence of mouse leukemia in animal experiments. Through the further experiment demonstrated that FTO by reducing some targets, such as RARA and ASB2 m6A levels, reduced its mRNA stability, ${ }^{32,33}$ and thus promoted the AML cells proliferation and survival, and suppressed alltrans-retinoic acid-induced AML cell differentiation and apoptosis, enhanced leukemia cancer gene-mediated cell transformation, thus promoted the occurrence of leukemia. ASB2 and RARA can inhibit myeloid leukemia cells' growth, promote their differentiation, and have antileukemia effects. The expression of FTO was significantly increased in breast cancer cells and tissues. Mechanically, it was found that the downstream target of m6A modification mediated by FTO was the pro-apoptotic gene BNIP3, which demethylation of BNIP3 messenger RNA m6A and induced its degradation, thus promoting the proliferation and inhibit apoptosis of breast cancer cells. ${ }^{34}$ Besides, FTO also promoted breast cancer cell proliferation and glycolysis through activation of the PI3K/ AKT signaling pathway, which had been demonstrated in patients with estrogen-receptor-positive breast cancer. ${ }^{35}$ Also, FTO was significantly up-regulated in estrogen-induced endometrial cancer, and the proliferation and invasion of endometrial cancer cells were enhanced by activation of PI3K/ AKT and AMPK signaling pathway. ${ }^{36}$ Activation of the PI3K/ AKT signaling pathway can lead to growth, proliferation, survival, cell cycle progression, and apoptosis inhibition of various tumor cells. ${ }^{37} \mathrm{Cui}$ et $\mathrm{al}^{38}$ demonstrated that m6A mRNA modification was significant for the growth of glioblastoma stem cells, and inhibition of demethylase FTO could inhibit the self-renewal of these tumor stem cells. By establishing a brain GBM mouse model and treating the mice with FTO inhibitor MA2, it was found that it could significantly suppress tumor progression and prolong the transplanted mice's survival time. Wu et $\mathrm{al}^{39}$ also confirmed the reliability of the above view through relevant studies. FTO expression was significantly elevated in tissues and cells of non-small cell lung cancer (NSCLC). Ding et $\mathrm{al}^{40}$ found that up-regulated FTO enhanced the proliferation and invasion of lung adenocarcinoma (LUAC) cells and inhibited their apoptosis through the activity of m6A demethylase. Another study found that
FTO promoted the proliferation and suppressed the apoptosis of lung squamous cell carcinoma (LUSC) by decreasing the level of myeloid zinc finger protein 1 (MZF1) m6A and increasing the stability of MZF1 mRNA. ${ }^{41}$ MZF1 has been shown to promote tumor progression in a variety of malignancies by regulating different targeted genes. In another study, FTO mediated the oncogenic effect of FTO in NSCLC cells via increasing the stability of ubiquitin-specific protease 7(USP7) mRNA through its demethylase enzyme activity. ${ }^{42}$ USP7 regulates substrate proteins' activity and stability and is involved in tumor inhibition, epigenetics, DNA damage response, and other pathways. ${ }^{43}$

The malignant progression of gastric cancer was significantly correlated with the regulatory factors of $\mathrm{m} 6 \mathrm{~A}$ RNA methylation. ${ }^{44} \mathrm{Xu}$ et al detected the protein and mRNA levels of FTO in 128 cases of gastric cancer tissues and found that FTO was highly expressed. ${ }^{45}$ Overexpression of FTO could promote the proliferation and migration of gastric cancer cell lines, while FTO's knockdown could achieve the opposite results. FTO was also upregulated in pancreatic cancer, and FTO knockdown decreased the proliferation of pancreatic cancer cells, suggesting that FTO was necessary for the malignant progression of pancreatic cancer. Besides, it was found that FTO enhanced the stability of MYC mRNA, enhanced the proliferation, and restrained the apoptosis of pancreatic cancer cells via interacting with the proto-oncogene MYC. ${ }^{46}$ The MYC was a key mediator in regulating the cell cycle. ${ }^{47}$ In a study of 60 patients with colorectal cancer, FTO was also found to be involved in the proproliferation and anti-apoptotic effects of miRNA-96 in colorectal cancer cells by blocking m6A methylation of AMP-activated protein kinase alpha2 (AMPKa2) and upregulated MYC expression. $^{48}$ Similarly, FTO regulated the modification of E2F1 and MYC transcripts through its demethylase activity and played a crucial role in promoting the proliferation and migration of cervical cancer cells. $^{49}$

\section{Impact of FTO on the Invasion and Migration of Cancers}

Invasion and metastasis play an essential role in tumor recurrence and poor prognosis. Histone deacetylase 3 (HDAC3), as a tumor-promoting factor, plays an oncogenic role in the development of gastric cancer. Yang et al found that HDAC3 reduced MYC m6A methylation in gastric cancer cells via regulated the $\mathrm{FTO} / \mathrm{m} 6 \mathrm{~A} / \mathrm{MYC}$ 
signaling pathway, thereby promoting MYC mRNA's stability thus enhanced the invasion and metastasis of gastric cancer cells. ${ }^{50}$ The results were also confirmed in mice in vivo. $\mathrm{Li}$ et $\mathrm{al}^{31}$ proved through in vitro and in vivo experiments that FTO promoted the invasion ability of AML cells and enhanced the cell transformation mediated by leukemia oncogenes, thus participating in the occurrence of AML. Besides, FTO enhanced the viability, proliferation, and transformation of AML cells by decreasing m6A modification in AML cells and increasing MYC/ CEBPA transcripts' stability. FTO was distinctly upregulated in HER2-positive breast cancer tissues and contributed to breast cancer cell invasion and migration through FTO/miR-181b-3p/ ARL5B signaling pathway. ${ }^{51}$ ADP ribosylation factor like GTPase 5B (ARL5B) is a small $\mathrm{G}$ protein existing in lysosomes, which promotes the movement of lysosomes and leads to their dispersion and aggregation in the periphery of cells, which is conducive to the progression of tumors. ${ }^{52,53}$

\section{FTO Showed Carcinogenic or Tumor Suppressive Characteristics in Tumors}

FTO, an oncogene, has been associated with poor survival in some malignancies. Shi et al found in the study of 1017 cases of NSCLC that FTO promoted the malignant progression of NSCLC by reducing the m6A level and activating the KRAS signaling pathway. ${ }^{54}$ Overexpression of FTO led to adverse clinical characteristics, and overall survival was worse when downregulation of the methyltransferase complex gene was associated with high expression of FTO. Up-regulated FTO was significantly associated with lymph node metastasis, poor differentiation, and poor prognosis in gastric cancer patients. ${ }^{45}$ Overexpression of FTO predicated lower overall survival (OS) and progression-free survival (PFS) in 450 cases of gastric cancer from the Tumor Genome Atlas (TCGA) database. However, TMA-IHC staining results showed that the lower the FTO protein level, the worse the overall survival of gastric cancer patients. ${ }^{55}$ The exact mechanism is still needed further research.

Similarly, FTO overexpression was significantly associated with tumor size, lymph node metastasis, TNM stage, and poor prognosis, which was confirmed in patients with HER2-positive breast cancer. ${ }^{51}$ Besides, high FTO expression in patients with type
I endometrial cancer indicated poor prognosis and early recurrence. ${ }^{56} \mathrm{Li}$ et al found that FTO was overexpressed in both hepatocellular carcinoma tissues and cells and promoted tumor growth via demethylation of PKM2, resulting in a lower survival rate. ${ }^{57}$ FTO was upregulated in cervical squamous cell carcinoma (CSCC) and enhanced the chemo-radiotherapy resistance probably via decreasing the level of m6A in $\beta$-catenin mRNA transcriptome and thereby increasing the activity of excision repair cross-complementation group 1 (ERCC1). ERCC1, a pivotal protein involved in DNA damage repair, plays a predictive role in the application of tumor chemotherapeutic agents. ${ }^{58}$ Furthermore, the prognosis of FTO on overall survival depended on the expression of $\beta$-catenin in human CSCC tissues (Table 1, Figure 2). ${ }^{59}$

However, as a tumor suppressor gene, FTO expression was significantly down-regulated in some tumors. FTO had a protective function in hepatocellular carcinoma (HCC). ${ }^{60}$ The down-regulation of FTO was correlated with tumor size, metastasis, and vascular invasion, which was associated with poor prognosis. $^{61}$ The expression of FTO was inhibited in clear cell renal cell carcinoma (ccRCC) tissues. FTO enhanced the anti-tumorigenic characters of ccRCC partly through reducing m6A levels of PPARg coactivators (PGC)-1 $\alpha$ mRNA transcripts and in turn, increased the expression of PGC-1 $\alpha$. In an analysis of 500 patients with ccRCC in The Cancer Genome Atlas (TCGA), low FTO expression predicted a worse prognosis. ${ }^{62}$ FTO suppressed the self-renewal of ovarian cancer stem cells through its demethylase activity and suppressed tumor progression by inhibiting the cAMP signaling pathway. ${ }^{63}$ Additionally, the specific mechanism and biological function are still unclear, and more studies are needed for further verification.

\section{Effects of FTO Inhibitors in Cancers}

Considering the tumorigenic role of FTO in various tumors, the research on FTO inhibitors is increasing gradually. Rhein, a natural product, was found to competitively bind to the active site of FTO and inhibit the demethylation activity of m6A. ${ }^{64}$ Compared with TKI monotherapy, combination with FTO inhibitor showed better efficacy in treating leukemia in mice. ${ }^{65}$ Recently, Huang et al designed two FTO inhibitors FB/FB23, that selectively suppress FTO's m6A demethylase activity. They significantly displayed the effect of antiproliferation and pro-apoptosis of AML cell lines in vitro and prolonged the survival of AML mouse models 


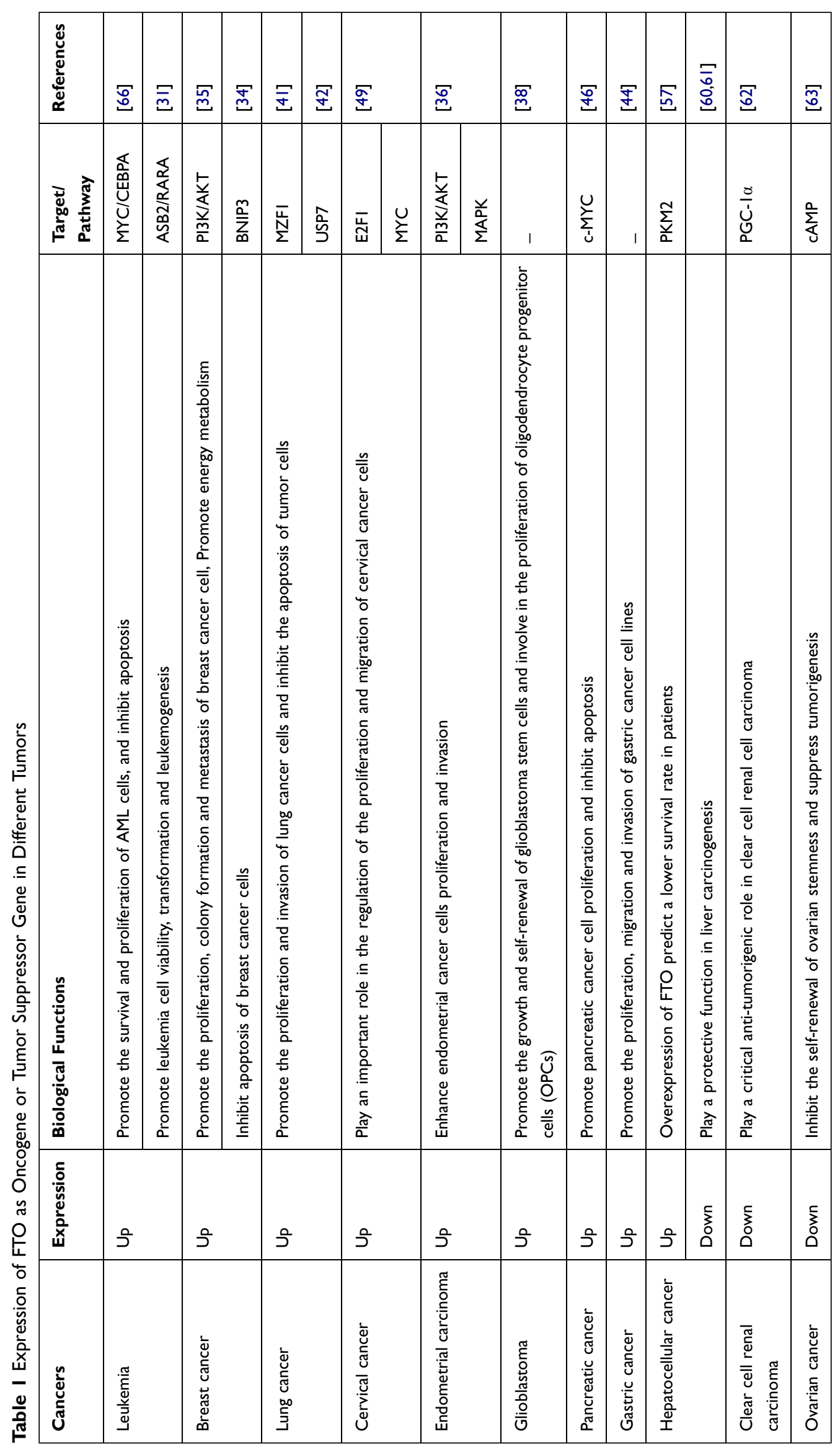




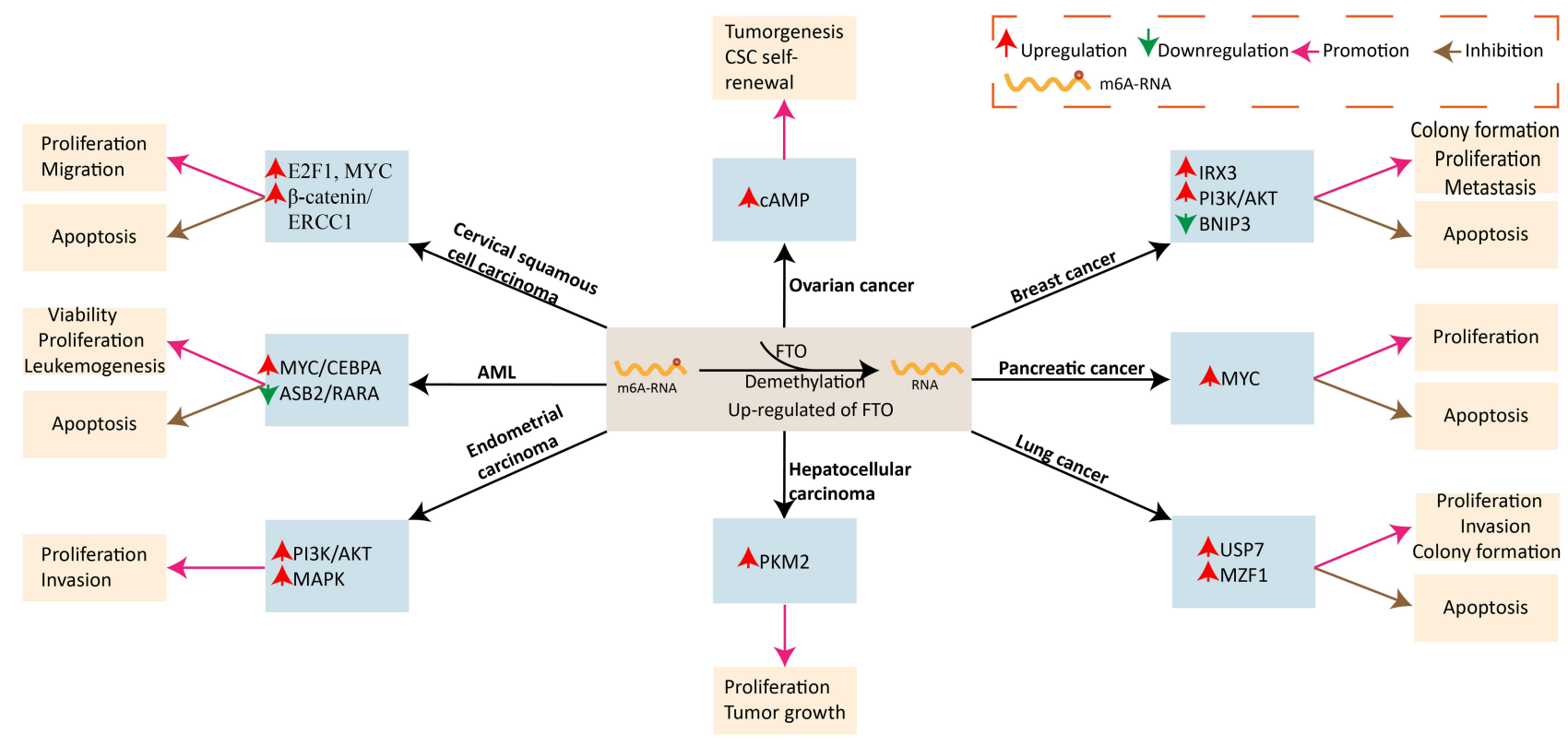

Figure 2 FTO overexpression in tumors and the regulation of some targets and signaling pathways.

in vivo. ${ }^{66} \mathrm{Su}$ et al first explored that R-2HG suppressed the activity of FTO and exerted anti-leukemia effects in vitro and in vivo via suppressing MYC/CEBPA signaling pathway by increasing the level of m6A mRNA. ${ }^{67} \mathrm{R}-2 \mathrm{HG}$ also showed solid antitumor activity in glioblastoma. Besides, Zheng et al developed a novel FTO inhibitor, later named MO-I-500, that selectively inhibited the m6A demethylation enzyme activity of FTO, increased intracellular m6A levels ${ }^{68}$ and was able to significantly inhibit the survival of triple-negative inflammatory breast cancer cell lines. ${ }^{69}$ As a non-steroidal anti-inflammatory drug, Meclofenamic acid (MA) is also a selective inhibitor of FTO, which can competitively combine with the FTO active site and suppress its demethylase activity. ${ }^{70}$ Compared with MA, the ethylester form of MA (MA2) significantly increased the level of m6A in cells, inhibited glioblastoma progression, prolonged the survival time of GSC-transplanted mice. ${ }^{38}$ In conclusion, a growing number of FTO inhibitors have shown positive therapeutic effects in animal models and may be ideal therapeutic targets for FTO overexpressed cancers in particular (Table 2).

\section{Conclusion and Prospect}

The FTO gene was initially thought to be involved in fat metabolism. With the discovery of the activity of
FTO as m6A demethylase, FTO as an oncogene or tumor suppressor gene has been studied continuously. FTO is involved in the occurrence and development of tumors by regulating some signaling pathways to promote tumor cell proliferation, transformation, activity enhancement, and stem cell self-renewal in different tumors. Besides, FTO also plays a critical role in the regulation of cancer stem cells. Therefore, FTO is being studied extensively as the most attractive new target for the therapy of cancers. The development of FTO inhibitors is also increasing and showing promising antitumor prospects in vitro and in vivo trials. Various inhibitors inhibiting FTO-mediated m6A demethylation have the potential to treat FTOoverexpressed cancers. In the future, it is hopeful that more FTO inhibitors will be validated in clinical trials and provide a safer and more effective treatment option for cancer patients.

However, FTO's exact role and molecular mechanisms in cancer are not fully understood, and there is still much work to be done. Therefore, further understanding of the molecular mechanisms and pathways that FTO regulates will provide essential insights into its role in regulating key oncogenic pathways and provide new and potential targets for the treatment of malignancies. 
Table 2 Mechanisms of Various Inhibitors by Inhibiting FTO-Mediated m6A Demethylation

\begin{tabular}{|l|l|l|l|}
\hline Inhibitors & Mechanisms & The Mechanisms of Anti-Cancer Effect & References \\
\hline Rhein & $\begin{array}{l}\text { Rhein competitively binds to the active site of } \\
\text { FTO and inhibits m6A demethylation }\end{array}$ & $\begin{array}{l}\text { Rhein combine with nilotinib is a more effective treatment for } \\
\text { leukemia in mice }\end{array}$ & [64] \\
\hline MA/MA2 & $\begin{array}{l}\text { MA/MA2 selectively inhibits m6A demethylation } \\
\text { of FTO }\end{array}$ & $\begin{array}{l}\text { MA2 significantly inhibits the growth and self-renewal of } \\
\text { glioblastoma (GSC) in vitro and prolongs the survival time of } \\
\text { GSC mice }\end{array}$ & [38,70] \\
\hline FB23/ & $\begin{array}{l}\text { FB23/FB23-2 directly binds to FTO and selectively } \\
\text { inhibit FTO's m6A demethylase activity }\end{array}$ & $\begin{array}{l}\text { FB23-2 inhibits the proliferation of human acute myeloid } \\
\text { leukemia (AML) cell lines and primary AML cells in vitro, and } \\
\text { promotes cell differentiation/apoptosis }\end{array}$ & [66] \\
\hline R-2HG & $\begin{array}{l}\text { R-2HG inhibits the activity of FTO } \\
\text { R-2HG inhibits the progression of leukemia and glioblastoma by } \\
\text { targeting the FTO/M6A/MYC/CEBPA signaling pathway }\end{array}$ & [67] \\
\hline MO-I-500 & $\begin{array}{l}\text { MO-I-500 is a mimic of 2-OG, which can } \\
\text { selectively inhibit FTO demethylation and increase } \\
\text { intracellular m6A levels }\end{array}$ & $\begin{array}{l}\text { MO-I-500 can significantly inhibit the growth of breast cancer } \\
\text { cells in vivo and in vitro }\end{array}$ & [68,69] \\
\hline
\end{tabular}

\section{Abbreviations}

FTO, Fat mass and obesity-associated gene; m6A, N6methyladenosine; SNPs, Single nucleotide polymorphisms; METTL14, Methyltransferase-like 14; METTL3, Methyltransferase-like 3; XPO2, Exportin 2; 3-Meu, 3-methyluracil; 3-Met, 3-methylthymine; AML, Acute myeloid leukemia; ASB2, Ankyrin-repeat SOCS box-containing protein 2; RARA, Retinoic acid receptor alpha; mRNA, Messenger RNA; PI3K/AKT, Phosphoinositide 3-kinases/protein kinase B; AMPK, Adenosine monophosphate-activated protein kinase; BNIP3, BCL2 Interacting Protein 3; NSCLC, Nonsmall cell lung cancer; LUAC, Lung adenocarcinoma; LUSC, Lung squamous cell carcinoma; MZF1, Myeloid zinc finger protein 1; USP7, Ubiquitin-specific protease 7; AMPK $\alpha 2$, AMP-activated protein kinase alpha2; HDAC3, Histone deacetylase 3; R-2HG, R-2-hydroxyglutarate; PKM2, Pyruvate kinase; CSCC, Cervical squamous cell carcinoma; ERCC1, Excision repair cross-complementation group 1; HCC, Hepatocellular carcinoma; ccRCC, Clear cell renal cell carcinoma; MA, Meclofenamic acid; CEBPA, CCAAT enhancerbinding protein alpha.

\section{Data Sharing Statement}

All data and material during this research are included in the published article.

\section{Author Contributions}

All authors made substantial contributions to conception and design, acquisition of data, or analysis and interpretation of data; took part in drafting the article or revising it critically for important intellectual content; agreed to submit to the current journal; gave final approval of the version to be published; and agree to be accountable for all aspects of the work.

\section{Funding}

This work was supported by the Shandong Provincial Natural Science Foundation (grant no. ZR2020MH204), the 19th batch of science and technology innovation development plan of Jinan in 2020 (Clinical medicine science and technology innovation plan, grant no.202019032), and the second group of science and technology projects of Jinan Health Committee (grant no. 2020-3-15).

\section{Disclosure}

The authors report no conflicts of interest in this work.

\section{References}

1. Peters T, Ausmeier K, Rüther U. Cloning of Fatso (Fto), a novel gene deleted by the Fused toes (Ft) mouse mutation. Mammal Genome. 1999;10(10):983-986. doi:10.1007/s003359901144

2. Dina C, Meyre D, Gallina S, et al. Variation in FTO contributes to childhood obesity and severe adult obesity. Nat Genet. 2007;39 (6):724-726. doi:10.1038/ng2048

3. Yang J, Loos RJ, Powell JE, et al. FTO genotype is associated with phenotypic variability of body mass index. Nature. 2012;490 (7419):267-272. doi:10.1038/nature11401

4. Anselme I, Laclef C, Lanaud M, Rüther U, Schneider-Maunoury S. Defects in brain patterning and head morphogenesis in the mouse mutant Fused toes. Dev Biol. 2007;304(1):208-220. doi:10.1016/j. ydbio.2006.12.025

5. Frayling TM, Timpson NJ, Weedon MN, et al. A common variant in the FTO gene is associated with body mass index and predisposes to childhood and adult obesity. Science (New York, NY). 2007;316 (5826):889-894. doi:10.1126/science.1141634 
6. Robbens S, Rouzé P, Cock JM, Spring J, Worden AZ, Van de Peer Y. The FTO gene, implicated in human obesity, is found only in vertebrates and marine algae. J Mol Evol. 2008;66(1):80-84. doi:10.1007/ s00239-007-9059-z

7. Gulati P, Avezov E, Ma M, et al. Fat mass and obesity-related (FTO) shuttles between the nucleus and cytoplasm. Biosci Rep. 2014;34(5): e00144. doi:10.1042/bsr20140111

8. Gerken T, Girard CA, Tung YC, et al. The obesity-associated FTO gene encodes a 2-oxoglutarate-dependent nucleic acid demethylase. Science (New York, NY). 2007;318(5855):1469-1472. doi:10.1126/ science. 1151710

9. Jia G, Yang CG, Yang $\mathrm{S}$, et al. Oxidative demethylation of 3-methylthymine and 3-methyluracil in single-stranded DNA and RNA by mouse and human FTO. FEBS Lett. 2008;582(2324):3313-3319. doi:10.1016/j.febslet.2008.08.019

10. Han Z, Niu T, Chang J, et al. Crystal structure of the FTO protein reveals basis for its substrate specificity. Nature. 2010;464 (7292):1205-1209. doi:10.1038/nature08921

11. Wang X, Lu Z, Gomez A, et al. N6-methyladenosine-dependent regulation of messenger RNA stability. Nature. 2014;505 (7481):117-120. doi:10.1038/nature12730

12. Liu N, Dai Q, Zheng G, He C, Parisien M, Pan T. N(6)methyladenosine-dependent RNA structural switches regulate RNA-protein interactions. Nature. 2015;518(7540):560-564. doi:10.1038/nature14234

13. Yang Y, Fan X, Mao M, et al. Extensive translation of circular RNAs driven by N(6)-methyladenosine. Cell Res. 2017;27(5):626-641. doi:10.1038/cr.2017.31

14. Jia G, Fu Y, Zhao X, et al. N6-methyladenosine in nuclear RNA is a major substrate of the obesity-associated FTO. Nat Chem Biol. 2011;7(12):885-887. doi:10.1038/nchembio.687

15. Zhao BS, Roundtree IA, He C. Post-transcriptional gene regulation by mRNA modifications. Nat Rev Mol Cell Biol. 2017;18(1):31-42. doi:10.1038/nrm.2016.132

16. Ping X-L, Sun B-F, Wang L, et al. Mammalian WTAP is a regulatory subunit of the RNA N6-methyladenosine methyltransferase. Cell Res. 2014;24(2):177-189. doi:10.1038/cr.2014.3

17. Liu J, Yue Y, Han D, et al. A METTL3-METTL14 complex mediates mammalian nuclear RNA N6-adenosine methylation. Nat Chem Biol. 2014;10(2):93-95. doi:10.1038/nchembio.1432

18. Yang G, Sun Z, Zhang N. Reshaping the role of m6A modification in cancer transcriptome: a review. Cancer Cell Int. 2020;20:353. doi:10.1186/s12935-020-01445-y

19. Wang X, Zhao BS, Roundtree IA, et al. N(6)-methyladenosine modulates messenger RNA translation efficiency. Cell. 2015;161 (6):1388-1399. doi:10.1016/j.cell.2015.05.014

20. Mauer J, Luo X, Blanjoie A, et al. Reversible methylation of $\mathrm{m}(6)$ $\mathrm{A}(\mathrm{m})$ in the $5^{\prime}$ cap controls mRNA stability. Nature. 2017;541 (7637):371-375. doi:10.1038/nature21022

21. Wei J, Liu F, Lu Z, et al. Differential m(6)A, m(6)A(m), and m(1)A demethylation mediated by FTO in the cell nucleus and cytoplasm. Mol Cell. 2018;71(6):973-985.e975. doi:10.1016/j.molcel.2018.08.011

22. Zhang X, Wei LH, Wang Y, et al. Structural insights into FTO's catalytic mechanism for the demethylation of multiple RNA substrates. Proc Natl Acad Sci USA. 2019;116(8):2919-2924. doi:10.1073/pnas.1820574116

23. Scuteri A, Sanna S, Chen WM, et al. Genome-wide association scan shows genetic variants in the FTO gene are associated with obesity-related traits. PLoS Genet. 2007;3(7):e115. doi:10.1371/journal.pgen.0030115

24. Karra E, O’Daly OG, Choudhury AI, et al. A link between FTO, ghrelin, and impaired brain food-cue responsivity. J Clin Invest. 2013;123(8):3539-3551. doi:10.1172/jci44403

25. Berulava T, Horsthemke B. The obesity-associated SNPs in intron 1 of the FTO gene affect primary transcript levels. EJHG. 2010;18 (9):1054-1056. doi:10.1038/ejhg.2010.71
26. Hernández-Caballero ME, Sierra-Ramírez JA. Single nucleotide polymorphisms of the FTO gene and cancer risk: an overview. Mol Biol Rep. 2015;42(3):699-704. doi:10.1007/s11033-014-3817-y

27. Kaklamani V, Yi N, Sadim M, et al. The role of the fat mass and obesity associated gene (FTO) in breast cancer risk. BMC Med Genet. 2011;12:52. doi:10.1186/1471-2350-12-52

28. Iles MM, Law MH, Stacey SN, et al. A variant in FTO shows association with melanoma risk not due to BMI. Nat Genet. 2013;45(4):428-432, 432e421. doi:10.1038/ng.2571

29. Huang X, Zhao J, Yang M, Li M, Zheng J. Association between FTO gene polymorphism (rs9939609 T/A) and cancer risk: a meta-analysis. Eur J Cancer Care (Engl). 2017;26(5):e12464. doi:10.1111/ecc. 12464

30. Tung YCL, Yeo GSH, O'Rahilly S, Coll AP. Obesity and FTO: changing focus at a complex locus. Cell Metab. 2014;20 (5):710-718. doi:10.1016/j.cmet.2014.09.010

31. Li Z, Weng H, Su R, et al. FTO plays an oncogenic role in acute myeloid leukemia as a N(6)-methyladenosine RNA demethylase. Cancer Cell. 2017;31(1):127-141. doi:10.1016/j.ccell.2016.11.017

32. Guibal FC, Moog-Lutz C, Smolewski P, et al. ASB-2 inhibits growth and promotes commitment in myeloid leukemia cells. J Biol Chem. 2002;277(1):218-224. doi:10.1074/jbc.M108476200

33. Glasow A, Prodromou N, Xu K, von Lindern M, Zelent A. Retinoids and myelomonocytic growth factors cooperatively activate RARA and induce human myeloid leukemia cell differentiation via MAP kinase pathways. Blood. 2005;105(1):341-349. doi:10.1182/blood2004-03-1074

34. Niu Y, Lin Z, Wan A, et al. RNA N6-methyladenosine demethylase FTO promotes breast tumor progression through inhibiting BNIP3. Mol Cancer. 2019;18(1):46. doi:10.1186/s12943-019-1004-4

35. Liu Y, Wang R, Zhang L, Li J, Lou K, Shi B. The lipid metabolism gene FTO influences breast cancer cell energy metabolism via the PI3K/AKT signaling pathway. Oncol Lett. 2017;13(6):4685-4690. doi:10.3892/o1.2017.6038

36. Zhang Z, Zhou D, Lai Y, et al. Estrogen induces endometrial cancer cell proliferation and invasion by regulating the fat mass and obesity-associated gene via PI3K/AKT and MAPK signaling pathways. Cancer Lett. 2012;319(1):89-97. doi:10.1016/j. canlet.2011.12.033

37. Noorolyai S, Shajari N, Baghbani E, Sadreddini S, Baradaran B. The relation between PI3K/AKT signalling pathway and cancer. Gene. 2019;698:120-128. doi:10.1016/j.gene.2019.02.076

38. Cui Q, Shi H, Ye P, et al. mA RNA methylation regulates the self-renewal and tumorigenesis of glioblastoma stem cells. Cell Rep. 2017;18(11):2622-2634. doi:10.1016/j.celrep.2017.02.059

39. Wu R, Li A, Sun B, et al. A novel mA reader Prrc2a controls oligodendroglial specification and myelination. Cell Res. 2019;29 (1):23-41. doi:10.1038/s41422-018-0113-8

40. Ding Y, Qi N, Wang K, et al. FTO facilitates lung adenocarcinoma cell progression by activating cell migration through mRNA demethylation. Onco Targets Ther. 2020;13:1461-1470. doi:10.2147/ott.S231914

41. Liu J, Ren D, Du Z, Wang H, Zhang H, Jin Y. m6 A demethylase FTO facilitates tumor progression in lung squamous cell carcinoma by regulating MZF1 expression. Biochem Biophys Res Commun. 2018;502(4):456-464. doi:10.1016/j.bbrc.2018.05.175

42. Li J, Han Y, Zhang H, et al. The m6A demethylase FTO promotes the growth of lung cancer cells by regulating the m6A level of USP7 mRNA. Biochem Biophys Res Commun. 2019;512(3):479-485. doi:10.1016/j.bbrc.2019.03.093

43. Bojagora A, Saridakis V. USP7 manipulation by viral proteins. Virus Res. 2020;286:198076. doi:10.1016/j.virusres.2020.198076

44. Su Y, Huang J, Hu J. mA RNA methylation regulators contribute to malignant progression and have clinical prognostic impact in gastric cancer. Front Oncol. 2019;9:1038. doi:10.3389/fonc.2019.01038 
45. Xu D, Shao W, Jiang Y, Wang X, Liu Y, Liu X. FTO expression is associated with the occurrence of gastric cancer and prognosis. Oncol Rep. 2017;38(4):2285-2292. doi:10.3892/or.2017.5904

46. Tang X, Liu S, Chen D, Zhao Z, Zhou J. The role of the fat mass and obesity-associated protein in the proliferation of pancreatic cancer cells. Oncol Lett. 2019;17(2):2473-2478. doi:10.3892/ol.2018.9873

47. Hsieh AL, Walton ZE, Altman BJ, Stine ZE, Dang CV. MYC and metabolism on the path to cancer. Semin Cell Dev Biol. 2015;43:11-21. doi:10.1016/j.semcdb.2015.08.003

48. Yue C, Chen J, Li Z, Li L, Chen J, Guo Y. microRNA-96 promotes occurrence and progression of colorectal cancer via regulation of the AMPKo2-FTO-m6A/MYC axis. J Exp Clin Cancer Res. 2020;39 (1):240. doi:10.1186/s13046-020-01731-7

49. Zou D, Dong L, Li C, Yin Z, Rao S, Zhou Q. The m6A eraser FTO facilitates proliferation and migration of human cervical cancer cells. Cancer Cell Int. 2019;19:321. doi:10.1186/s12935-019-1045-1

50. Yang Z, Jiang X, Zhang Z, et al. HDAC3-dependent transcriptional repression of FOXA2 regulates FTO/m6A/MYC signaling to contribute to the development of gastric cancer. Cancer Gene Ther. 2021;28(1-2):141-155. doi:10.1038/s41417-020-0193-8

51. Xu Y, Ye S, Zhang N, et al. The FTO/miR-181b-3p/ARL5B signaling pathway regulates cell migration and invasion in breast cancer. Cancer Commun. 2020;40(10):484-500. doi:10.1002/cac2.12075

52. Garg S, Sharma M, Ung C, et al. Lysosomal trafficking, antigen presentation, and microbial killing are controlled by the Arf-like GTPase Arl8b. Immunity. 2011;35(2):182-193. doi:10.1016/j. immuni.2011.06.009

53. Dykes SS, Gray AL, Coleman DT, et al. The Arf-like GTPase Arl8b is essential for three-dimensional invasive growth of prostate cancer in vitro and xenograft formation and growth in vivo. Oncotarget. 2016;7(21):31037-31052. doi:10.18632/oncotarget.8832

54. Shi H, Zhao J, Han L, et al. Retrospective study of gene signatures and prognostic value of $\mathrm{m} 6 \mathrm{~A}$ regulatory factor in non-small cell lung cancer using TCGA database and the verification of FTO. Aging. 2020;12(17):17022-17037. doi:10.18632/aging.103622

55. Li Y, Zheng D, Wang F, Xu Y, Yu H, Zhang H. Expression of demethylase genes, FTO and ALKBH1, is associated with prognosis of gastric cancer. Dig Dis Sci. 2019;64(6):1503-1513. doi:10.1007/ s10620-018-5452-2

56. Zhu Y, Shen J, Gao L, Feng Y. Estrogen promotes fat mass and obesity-associated protein nuclear localization and enhances endometrial cancer cell proliferation via the mTOR signaling pathway. Oncol Rep. 2016;35(4):2391-2397. doi:10.3892/or.2016.4613

57. Li J, Zhu L, Shi Y, Liu J, Lin L, Chen X. m6A demethylase FTO promotes hepatocellular carcinoma tumorigenesis via mediating PKM2 demethylation. Am J Transl Res. 2019;11(9):6084-6092.
58. Formica V, Doldo E, Antonetti FR, et al. Biological and predictive role of ERCC1 polymorphisms in cancer. Crit Rev Oncol Hematol. 2017;111:133-143. doi:10.1016/j.critrevonc.2017.01.016

59. Zhou S, Bai ZL, Xia D, et al. FTO regulates the chemo-radiotherapy resistance of cervical squamous cell carcinoma (CSCC) by targeting $\beta$-catenin through mRNA demethylation. Mol Carcinog. 2018;57 (5):590-597. doi:10.1002/mc.22782

60. Mittenbühler MJ, Saedler K, Nolte H, et al. Hepatic FTO is dispensable for the regulation of metabolism but counteracts HCC development in vivo. Mol Metabol. 2020;42:101085. doi:10.1016/j. molmet.2020.101085

61. Zhao Y, You S, Yu YQ, et al. Decreased nuclear expression of FTO in human primary hepatocellular carcinoma is associated with poor prognosis. Int J Clin Exp Pathol. 2019;12(9):3376-3383.

62. Zhuang C, Zhuang C, Luo X, et al. N6-methyladenosine demethylase FTO suppresses clear cell renal cell carcinoma through a novel FTOPGC-1 $\alpha$ signalling axis. $J$ Cell Mol Med. 2019;23(3):2163-2173. doi:10.1111/jcmm. 14128

63. Huang $\mathrm{H}$, Wang $\mathrm{Y}$, Kandpal $\mathrm{M}$, et al. FTO-dependent $\mathrm{N}$ (6)-methyladenosine modifications inhibit ovarian cancer stem cell self-renewal by blocking cAMP signaling. Cancer Res. 2020;80 (16):3200-3214. doi:10.1158/0008-5472.Can-19-4044

64. Chen B, Ye F, Yu L, et al. Development of cell-active N6-methyladenosine RNA demethylase FTO inhibitor. $\mathrm{J} \mathrm{Am} \mathrm{Chem}$ Soc. 2012;134(43):17963-17971. doi:10.1021/ja3064149

65. Yan F, Al-Kali A, Zhang Z, et al. A dynamic N(6)-methyladenosine methylome regulates intrinsic and acquired resistance to tyrosine kinase inhibitors. Cell Res. 2018;28(11):1062-1076. doi:10.1038/ s41422-018-0097-4

66. Huang Y, Su R, Sheng Y, et al. Small-molecule targeting of oncogenic FTO demethylase in acute myeloid leukemia. Cancer Cell. 2019;35(4):677-691.e610. doi:10.1016/j.ccell.2019.03.006

67. Su R, Dong L, Li C, et al. R-2HG exhibits anti-tumor activity by targeting FTO/m(6)A/MYC/CEBPA signaling. Cell. 2018;172(12):90-105.e123. doi:10.1016/j.cell.2017.11.031

68. Zheng G, Cox T, Tribbey L, et al. Synthesis of a FTO inhibitor with anticonvulsant activity. ACS Chem Neurosci. 2014;5(8):658-665. doi:10.1021/cn500042t

69. Singh B, Kinne HE, Milligan RD, Washburn LJ, Olsen M, Lucci A. Important role of FTO in the survival of rare panresistant triple-negative inflammatory breast cancer cells facing a severe metabolic challenge. PLoS One. 2016;11(7):e0159072. doi:10.1371/journal.pone. 0159072

70. Huang Y, Yan J, Li Q, et al. Meclofenamic acid selectively inhibits FTO demethylation of m6A over ALKBH5. Nucleic Acids Res. 2015;43(1):373-384. doi:10.1093/nar/gku1276
OncoTargets and Therapy

\section{Publish your work in this journal}

OncoTargets and Therapy is an international, peer-reviewed, open access journal focusing on the pathological basis of all cancers, potential targets for therapy and treatment protocols employed to improve the management of cancer patients. The journal also focuses on the impact of management programs and new therapeutic agents and protocols on patient perspectives such as quality of life, adherence and satisfaction. The manuscript management system is completely online and includes a very quick and fair peer-review system, which is all easy to use. Visit http://www.dovepress.com/ testimonials.php to read real quotes from published authors. 